\title{
Microgrid Design using Folded $P$ - $f$ Droop and New Grid Interface Unit to Minimize the Need for Communication
}

\author{
Xiaozuo Huang, Dong Chen, and Lie Xu
}

\begin{abstract}
A new control scheme is proposed for microgrids to enable seamless switching between islanded mode and gridconnected mode in this paper. Utilizing a specially designed interface unit as the interface device, a systematic control strategy is proposed to enable a smooth and autonomous transition from grid-connected to islanded and vice-versa. With the proposed scheme, the need for high-speed communication is eliminated despite of the change of operational mode. To make this happen, a folded droop scheme is proposed to enable autonomous frequency support for paralleled converters in the microgrid and a coordinating strategy is then proposed to enable autonomous resynchronization with the main grid as well. The control scheme proposed are validated with a benchmark microgrid model of parallel- connected converters using MATLAB/SIMULINK. The results show that the proposed control can enable autonomous smooth transitions between modes with reduced power rating in the interface converter unit.
\end{abstract}

Index Terms-Microgrid, Seamless transition, Resynchronization, Back-to-back Converter, Islanded mode, Gridconnected mode, Autonomous operation.

\section{INTRODUCTION}

A ddressing the big picture of Paris Agreement, the Nationally Determined Contribution (NDC) of EU plans to reduce $\mathrm{CO}_{2}$ emissions by $40 \%$ before 2030 . Undertaking the challenges of generating non-nuclear and fossil fuel free energy, microgrids (MGs) are adopting a growing employment of power electronics devices which aims to fulfill power conversion and essential control. To provide reliable power supplies to end users with significant intermittent renewable energy generation, the design of MGs is expected to adapt to both grid-connected and islanded conditions. Although control of microgrids has been intensively investigated in the past decade, a smooth transition scheme with reasonable cost and reliability remains a challenge for a microgrid of multiple converters.

Given a relatively stiff voltage in place during gridconnected condition, grid-following control with closed current/power loops is commonly used for the interface converter of distributed energy resources (DERs) [1] but such strategy alone cannot balance the power when the mains is lost. Therefore, intensive research has been conducted in exploring the control of power converters in an islanded MG, where grid forming control is used [2]. Applying voltage/frequency regulation to the outer loop of DERs, cascaded control strategy is usually used to establish a voltage source for a standalone MG [3]. However, such strong voltage regulation is not entirely compatible for grid-connected condition [4]. Thus, a consequent challenge is how to accommodate grid-connected and islanded conditions in one decentralized control scheme when the operational condition is subject to change. Mode switching strategies between grid following strategy and droopbased grid forming strategy is therefore suggested [5], but its robustness is challenged by the smoothness and reliability of mode switching. The major challenge is associated with two aspects: the triggering scheme of mode switching and the need for communications. Despite of many proposed islanding detection schemes with both passive and/or active approaches [4][6], it is still a challenge to correctly trigger islanding operation without compromising the performance in power quality and reliability [7]. When a participating DER is located far from the Point of Connection (PoC) to the mains, high bandwidth communication is required for islanding and resynchronization operations [8][9]. The inclusion of communication in a high bandwidth control loop [1] [10][11][12] will not only increase the cost but also the risk of operational failure, as the intermittency of communication is inevitable over time.

To improve the islanding operation capability, control strategies of multiple cascaded loops are suggested [9] [13] [14]. By adding power regulation out of the voltage loops and Phase Locked Loop (PLL) feedback to regulate local voltage vector, such control can adapt to a fast-changing grid condition. As a result, it does not need communications among DERs for islanding transition; However, it still requires fast communication to re-synchronize with the mains for reconnection transition, especially when the DER is not closely located beside the PoC [15].

As a more reliable solution, back-to-back (BTB) converter can be employed as a $\mathrm{MG}$ interface to achieve seamless transition with local detection, but this will significantly add up to the losses during grid-connected operation. Since a large transient could still be introduced during islanding transition [1], a secondary control is usually designed to restore the frequency and voltage, but again fast communication is required [16][17][18][19] . Other alternatives such as cascaded control and virtual synchronous machine (VSM) control have been 
proposed but their performances against a major transient transition is still to be improved [20] [21].In this paper, a novel microgrid operational scheme is proposed. Unlike those schemes of non-linear mode switching [22][23], the proposed scheme can enable seamless power supply autonomously; at the meantime, it preserves the smooth transitional performance of BTB scheme [24] with reduced capital cost and improved steady state performance. The proposed scheme further benefits from improve robustness by eliminating the need for fast communications.

The rest of the paper is organized as follows. Section II outlines the system configuration and different operation modes. The proposed scheme is described in Section III and case studies are presented in Section IV to verify the proposed control strategies. Finally, Section V draws conclusions.

\section{SYSTEM CONFIGURATIONS AND OPERATION MODES}

\section{A. System Configuration}

To demonstrate and validate the proposed control scheme, a benchmark MG system is set up as Fig. 1 shows. As shown, the proposed MG system mainly consists of three parts: converter interfaced DER units, loads and a grid Interface Unit (IU). The system can include both dispatchable and non-dispatchable DGs though the specific DERs considered in the paper are those dispatchable ones, for example, battery energy storage system or electric vehicle chargers can adopt this strategy.

1. DERs are modeled as the interfaced VSCs between deterministic energy sources and the MG, assuming that the energy capacities are adequate for the required operations. To demonstrate the possible dynamic interaction between DERs, two DERs are considered using average VSC models.

2. A grid Interface Unit (IU) configuration is proposed in this paper to assist the reconnection of the MG to AC grid. It consists of a set of BTB VSCs (IU-VSC1 and 2) in parallel with a circuit breaker (IU-CB).

3. To simplify the system, resistive and inductive loads are considered.

4. Filter capacitors are placed at each terminal of the DERs within the MG, as shown in Fig. 1. The power lines between DERs and loads are modeled as resistive and inductive impedances, as the distributed capacitances of the lines are negligible comparing to DER filter capacitances. The leakage reactance of VSC transformers are modeled as a part of the power line impedances.

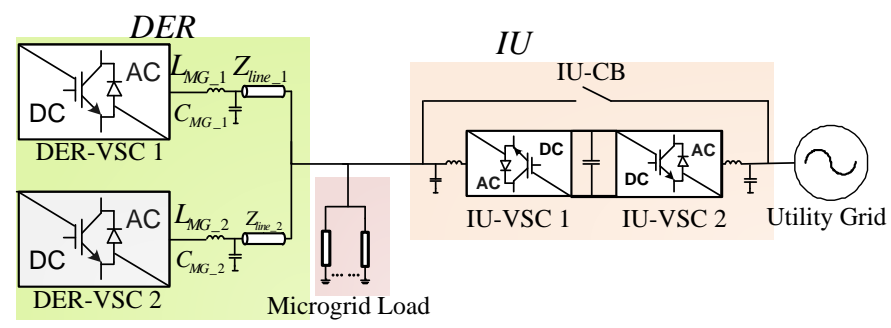

Fig. 1. System configuration.

\section{B. Operation Modes in Steady State}

Grid-connected mode corresponds to the situation when the MG is connected to the utility grid. The MG can be connected to the mains through either IU-CB or the IU-VSCs. To minimize the semiconductor losses, the connection is via IU$\mathrm{CB}$ at steady state; whereas IU-VSC1 and 2 are only used during transitions between operational modes. Under gridconnected mode, DERs deliver their powers on their own merits and power balancing within the $\mathrm{MG}$ is accommodated by the main grid.

The islanded mode refers to the condition when the MG is disconnected from the main grid. Thus, MG voltage is formed by DERs and the power flow within the MG must be balanced locally. During this mode, the net load within the MG is autonomously shared among DERs.

\section{Transitional operation modes}

Transitional operation modes refer to the condition when the MG is in the process of transferring from grid-connected mode to islanded or vice versa. Thus, it can be divided into two transitional conditions as follows.

\section{1) Islanding transition.}

Islanding transition is defined as the process when the $\mathrm{MG}$ is transferring from grid-connected to islanded. Such transitions can be divided into two types: planned islanding and unplanned islanding. In this paper, planned islanding is proposed to use IU-VSCs to smoothly take over all the power flow from IU-CB and then taper off it controllably, avoiding any large transient to the MG. In contrast, an unplanned islanding happens when the external voltage source of the utility is lost by opening IUCB. During this transition, DERs are expected to take over the control of MG voltage immediately after opening IU-CB. Meanwhile, the transient currents of DERs must be capped at their ratings, respectively.

\section{2) Reconnection transition.}

Reconnection transition refers to the situation that MG is transferring from islanded to grid-connected operation. This involves the following steps that are facilitated by the proposed IU unit: (1) Synchronize the MG voltage (instantaneous angle, magnitude) with utility grid using IU-VSCs; (2) Connecting to mains by closing IU-CB; (3) De-load the power transfer via IU-VSCs down to zero; (4) Block the IU-VSCs.

Similar to planned islanding transition, this transitional process does not have to be triggered immediately after the utility grid is ready for connection. Since the MG voltage is well-supported by DERs during the transition, a slight delay will not affect the reliability of power supply within the MG.

\section{System Level Operational Strategy}

The proposed transitional processes between modes are demonstrated in Fig. 2. The transitions are carried out by coordinating the operation of the three types of devices, i.e., DERs, IU-VSCs and IU-CB. In islanded mode, DERs work at grid forming state to establish the voltage of $\mathrm{MG}$ and automatically share the power supply to local loads. As is fore cited, the BTB IU-VSCs are blocked and IU-CB is open in islanded mode. Under grid-connected mode, DERs deliver powers on their own merits, IU-VSCs are blocked and IU-CB is closed.

An intermediate state for transferring from islanded mode to grid-connected is defined as "resynchronization". During 
resynchronization, DERs are still actively sustaining the voltage of MG, but IU-VSCs start to drive the angle and magnitude of MG voltage towards the mains. This means that during this process, the MG voltage is actively formed by the co-operation of DERs and IU-VSC1 while IU-CB remains open. Once the instantaneous angles and voltages across both sides of the IU-CB consistently match in pairs, IU-CB is closed, which initiates the transition between resynchronization and gridconnected mode. By gradually de-loading IU-VSC1's active and reactive power to zero, the power flows through IU-CB increases accordingly. IU-VSCs can be subsequently blocked for a smooth transition and the MG starts to operate under gridconnected mode.

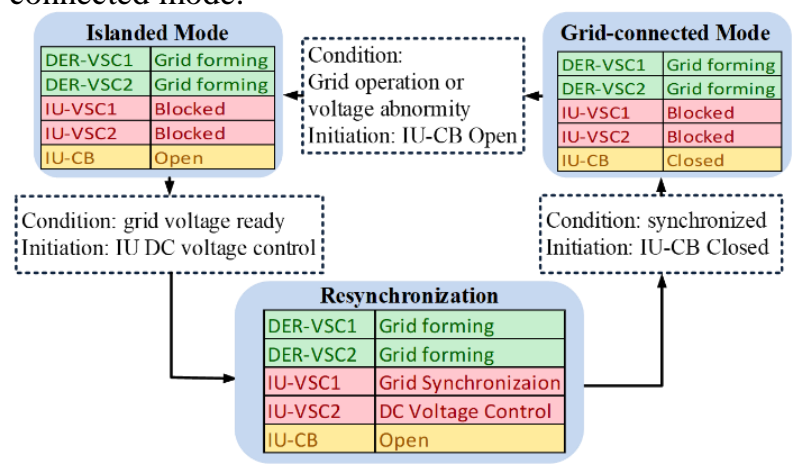

Fig. 2. Operation process and states.
The transient from grid-connect to islanded mode can be intentional (i.e. planned) for network operation or unintentional due to grid abnormality. For intentional transition, the fore cited re-conection process is reversed. With a planned pre-operation net load, the IU-VSCs takes over the net load after IU-CB is open and gradually release it before they are blocked. Thus, the impact of sudden pick-up of net load by DERs is minimized. For unintentional transition, IU-CB opens and DERs immediately take over the voltage control to operate in islanded mode.

\section{CONTROL SCHEMES OF DERS AND IU}

In this section, the control scheme of DERs is presented in the first place. The operational scheme of IU is the introduced as part of the cooperation between DERs and IU to achieve previously described seamless transitions.

\section{A. Control of Deterministic DERs}

In order to ensure DERs can fulfil functions of current regulation during transients, autonomous synchronous voltage regulation during islanded operation and power control during grid connection, multiple closed loops of current, voltage and power control are integrated in a cascaded manner, which is demonstrate by Fig. 3 .

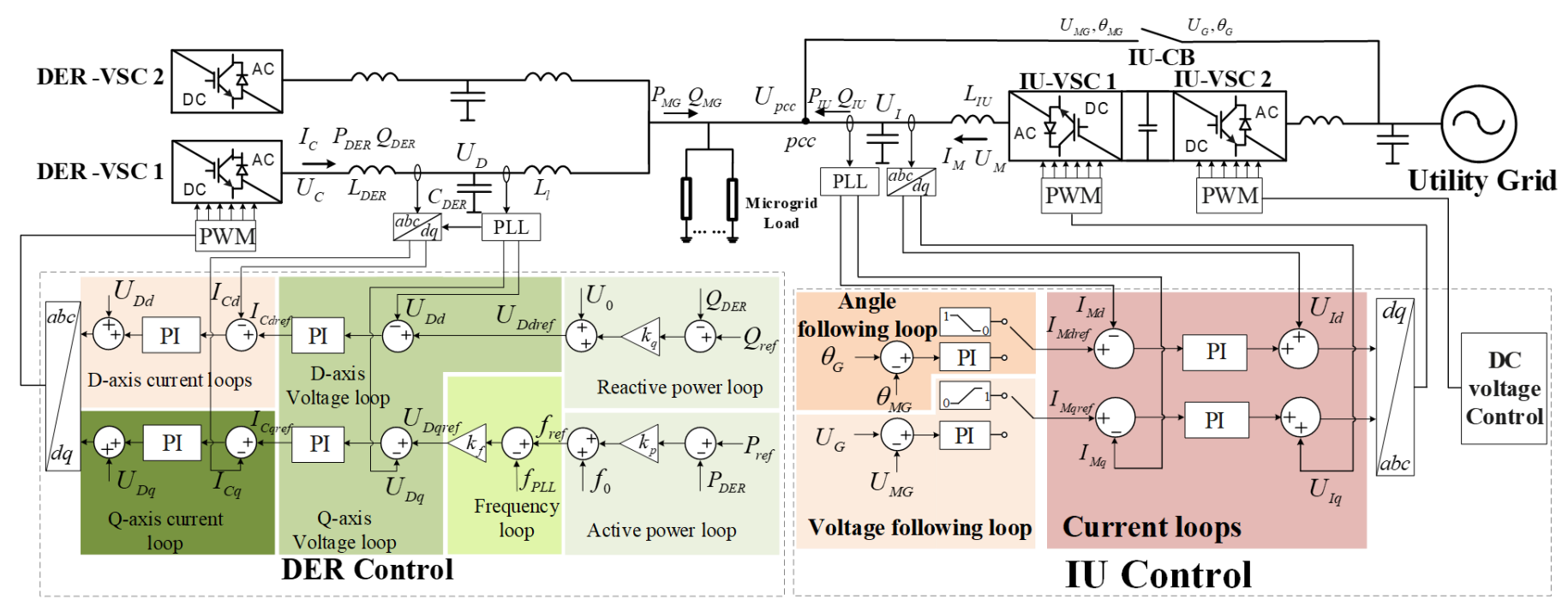

Fig. 3. Control of the proposed system.

Classical double-loop vector control of current and voltage with synchronous reference frame are employed as the inner loops [25][26][27]. To ensure unified control structure for the DERs during both grid-connect and islanded mode, additional outer active power-frequency droop and reactive power-voltage droop are implemented together with a frequency loop [13] [28][29].

As is shown in Fig. 3, under grid-connected operation, the outmost loops of active power and reactive power (feedback as $Q_{D E R}$ and $P_{D E R}$, respectively) actively dispatch the output power by adjusting the outputs, which are orders of capacitor voltage magnitude and frequency (change of power angle), in respect to the mains. All the rest of the loops can be considered as inner process to realize the power control. The functioning of such outmost loops relies on the prerequisite of an independent voltage support other than DER, which is by the mains.

However, when the mains are lost in an island, the microgrid is formed by the collective control among DERs. The powers of DERs are not deterministically dispatched but autonomously shared among voltage and frequency loops of DERs to accommodate the local net load. In this condition, the outmost power loops cannot function deterministically (as the microgrid voltage angle and magnitude are determined by the closed loops of voltage and frequencies) but only offset the reference values of frequency and voltage loops. This function shift of DER autonomously takes place immediately the mains are lost. Regardless of planned or unplanned transitions, the control structure of DERs remains unchanged. 


\section{B. Control of IU}

IU is designed to enable smooth transitions with decentralized control. During grid-connected operation as presented in section II, the CB of IU is closed, with the VSCs blocked. During islanded mode, the CB open, and the VSCs blocked.

Seamless synchronization transition is achieved by creating an interim state to synchronize the voltages across the $\mathrm{CB}[30]$. In this interim state, IU-VSC1 is initiated to regulate the voltage and frequency on the MG side to follow the utility voltage; whereas the DC link voltage of the IU-VSCs can be maintained by the grid-connected IU-VSC2 in the IU using cascaded control [26][27][28][31]. In this paper, the employed converter control strategy is demonstrated in Fig. 3.

To create a smooth transition for the MG, it is important that voltage magnitude, instantaneous angle of the MG are closely following the mains across the IU-CB simultaneously. This is to limit the transient current after the closure of IU-CB.

As previously described and shown in Fig. 3, for each DER, an active power loop is employed for power sharing and a frequency deviation $\Delta f$ will be produced by the arbitrary controller. For the $i^{\text {th }} \mathrm{DER}$, there is

$$
\Delta f=f_{M G}-f_{0}=K_{p i}\left(P_{D E R i}^{*}-P_{D E R i}\right)
$$

where $P_{D E R i}^{*}$ and $P_{D E R i}$ i are the set points and feedback of active power for the $i^{\text {th }}$ DER, respectively; $f_{M G}$ and $f_{0}$ the feedback and set point of MG frequency, respectively; $K_{p i}$ is the droop coefficient.

At steady state, the aggregated active power generation by the DERs equals to the aggregated load; the frequencies at the DER terminals are identical. Thus, before synchronization, the relationship between DER active power and MG frequency is given as

$$
\sum_{i=1}^{n} P_{D E R i}^{*}-P_{\text {Load }}=\left(f_{M G}-f_{0}\right) \sum_{i=1}^{n} \frac{1}{K_{p i}}
$$

where $n$ is the number of DERs ( $\mathrm{n}=2$ in this paper).

Therefore, the primary means to regulate the frequency and angle of the MG is via active power control of the IU-VSCs. Preserving the autonomous control of DERs, the frequency adjustment by IU-VSC can be carried out based on the following equation as

$$
\sum_{i=1}^{n} P_{D E R i}^{*}-P_{\text {Load }}+P_{I U}=\left(f_{\text {grid }}-f_{0}\right) \sum_{i=1}^{n} 1 / K_{p i}
$$

where $P_{I U}$ is the active power supplied by IU-VSC1 to the MG and $f_{\text {grid }}$ the frequency of the mains. Thus, the required power capacity of an IU-VSCs is

$$
P_{I U_{-} V S C 1}=|\Delta f| \sum_{i=1}^{n} 1 / K_{p i}
$$

where $|\Delta f|$ is the frequency deviation of the MG from the mains prior to re-synchronization.

To resynchronize MG with the mains, an angle following loop is developed as Fig. 3 shows. As seen, the error between the instant angles of the main grid and MG is regulated through a PI controller producing a reference active current order for IU-VSC1.

To mitigate the voltage difference between the MG and the mains, a voltage following loop is developed and coordinate the $\mathrm{Q}-\mathrm{V}$ reactive power loops of DERs. The error between the instantaneous voltage magnitudes of MG and the mains is regulated through a PI controller. The controller produces a reference reactive current order of IU-VSC 1, which is also shown in Fig. 3.

\section{The reduction of IU Size and Droop Folding Scheme}

Based on the classical $p-f$ droop control [14][19], the essential capacity of active power for an IU to ensure resynchronization of frequency can be derived from (3) as

$$
P_{I U \_ \text {max }}=\left|\Delta f_{\text {max }}\right| \sum_{i=1}^{n} 1 / K_{p i}
$$

where $\Delta f_{\text {max }}$ refers to the maximum frequency deviation across IU-CB, that is, the frequency deviation between MG and the mains under the most adverse scenario.

According to (5), when the frequency deviation between MG and the mains is at its maximum, it requires the highest IU power during resynchronization. The maximum frequency can be obtained by:

$$
\left|\Delta f_{\text {max }}\right|=\left|\Delta f_{M G_{-} \max }\right|+\left|\Delta f_{\text {grid_max }}\right|
$$

where $\Delta f_{\mathrm{MG}_{-} \max }$ and $\Delta f_{\text {grid_max }}$ are the maximum frequency deviations from the nominal frequency, e.g. $50 \mathrm{~Hz}$, of the MG and utility grid, respectively. Since $\Delta f_{\text {grid_max }}$ is usually fixed by the grid code, e.g. $0.3 \mathrm{~Hz}$, the maximum frequency deviation is primarily determined by $\Delta f_{\mathrm{MG}_{-} \max }$. Considering (2), there is

$$
\left|\Delta f_{\mathrm{MG}_{-} \max }\right|=\frac{\max \left(\left|\sum_{i=1}^{n} P_{D E R i}^{*}-P_{\text {Load }}\right|\right)}{\sum_{i=1}^{n} 1 / K_{p i}}
$$

where " $\max (\mathrm{)})$ " refers to the maximum function. Combining (5)-(7) yields

$P_{I U \_ \text {max }}=\max \left(\sum_{i=1}^{n} P_{D E R i}^{*}-P_{\text {Load }}\right)+\left|\Delta f_{\text {grid_max }}\right| \sum_{i=1}^{n} \frac{1}{K_{p i}}$

Equation (8) implies that the size of IU is determined by the maximum power required during re-connection. The reduction of IU size is determined by the differences between generation reference values and operating loads, $\max \left(\sum_{i=1}^{n} P_{D E R i}^{*}-\right.$ $\left.P_{\text {Load }}\right)$, which theoretically can be anywhere between -2 to 2 p.u..

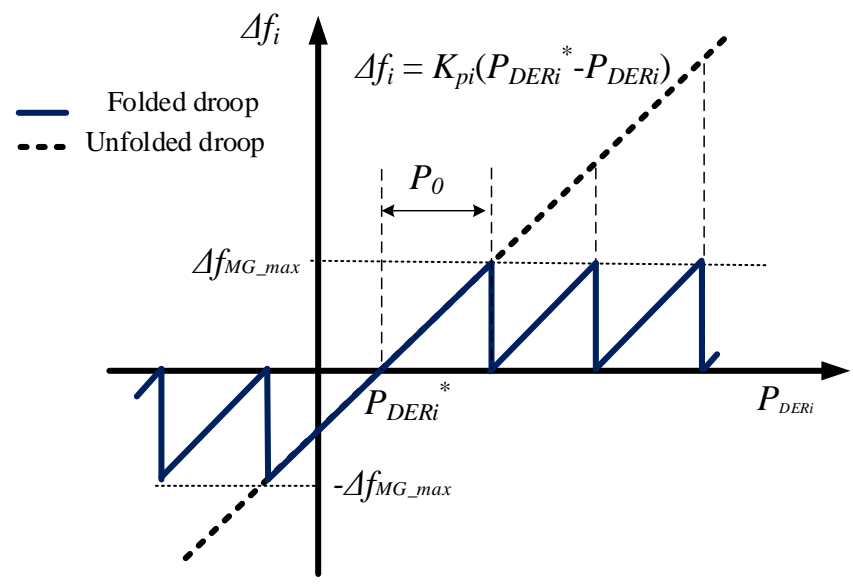

Fig. 4. Folded droop

Rearranging (1), there is

$$
P_{D E R i}^{*}=\Delta f / K_{p i}+P_{D E R i}
$$

At steady state of islanded mode, there is 


$$
\sum_{i=1}^{n} P_{D E R i}=P_{L o a d}
$$

substituting (9) (10) to (8), the change of IU size $\Delta P_{I U_{-} \max }$ can be calculated as

$$
P_{I U \_ \text {max }}=|\Delta f| \sum_{i=1}^{n}\left(\frac{1}{K_{p i}}\right)+\left|\Delta f_{\text {grid_max }}\right| \sum_{i=1}^{n} \frac{1}{K_{p i}}
$$

Given a pre-fixed value of grid frequency deviation, $P_{I U_{-} \text {max }}$ can be limited with a confined value of $M G$ frequency deviation $|\Delta f|$.

Considering (2) and as seen in Fig. 4, the original unfolded droop is given as $\Delta f_{i}=K_{p i}\left(P_{D E R i}^{*}-P_{D E R i}\right)$ and is represented by the dash line. During island operation when the frequency deviation is positive and moves upwards, the power reference value $\mathrm{P}_{\mathrm{i}}^{*}$ will be automatically deducted by $\Delta f_{M G_{-} \max } / \mathrm{K}_{\mathrm{pi}}$ if the magnitude of power continues to increase after the frequency deviation has reached the upper limit, $\Delta f_{M G_{-} \max }$. The same mechanism will be triggered every time the frequency limits is reached. As a result, the droop is "folded" as shown. Similarly, the droop is also folded symmetrically in the third quadrant every time when $-\Delta f_{M G_{-} \max }$ is reached. By folding the $P$ - $f$ droop line as shown in Fig. 4, the frequency can be enveloped within a predefined region, and hence, the rating of IU can be capped by a pre-defined value of maximum $\mathrm{MG}$ frequency deviation $\Delta f_{\max _{-} m g}$ as

$$
P_{I U \_ \text {max }}=\left(\left|\Delta f_{M G_{-} \max }\right|+\left|\Delta f_{\text {grid_max }}\right|\right) \sum_{i=1}^{n} \frac{1}{K_{p i}}
$$

The implementation of the folded droop is shown in Fig. 5.

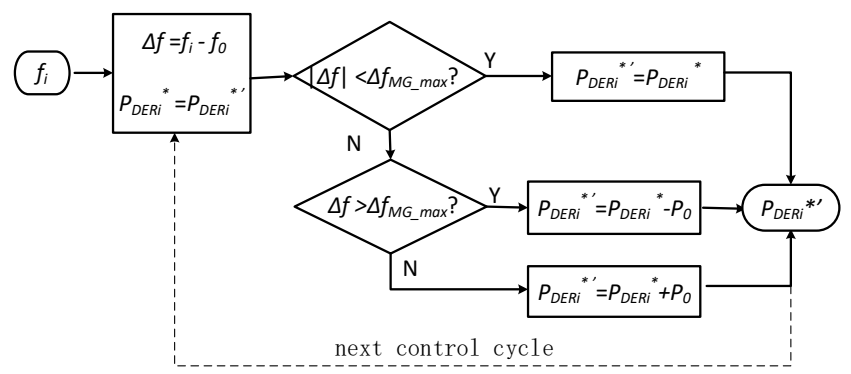

Fig. 5. Updated active power loop with folded droop.

As shown, at each control cycle, a judgment statement is used to evaluate the active power reference for the $i^{\text {th }}$ DER based on the measured frequency. Taking the $m^{\text {th }}$ control cycle as an example, $P_{D E R i}^{* \prime}(m)$ is given as

$$
P_{D E R i}^{* \prime}(m)=\left\{\begin{array}{cc}
P_{D E R i}^{* \prime}(m-1), & \Delta f_{i} \leq f_{\text {max } \_m g} \\
P_{D E R i}^{* \prime}(m-1)+P_{0}, & \Delta f_{i} \geq f_{\text {max }_{2} m g} \\
P_{D E R i}^{* \prime}(m-1)-P_{0}, & \Delta f_{i} \leq-f_{\text {max } \_m g}
\end{array}\right.
$$

where $P_{0}$ is a constant and as shown in Fig. 5, it is given as

$$
P_{0}=\frac{f_{\text {MG_max }}}{K_{p i}}
$$

Thus, by folding the DER power reference according to frequency and then cap the output frequency deviation, the proposed folded droop can be employed to update the active power loop reference in Fig. 3.

By substituting the operating frequency deviations $\left(\Delta f_{1}\right.$ and $\Delta f_{2}$ ) into (11), the change of minimum IU power to maintain synchronization in a specific reconnection operation can be determined as

$$
\Delta P_{I U_{-} \max }=\left(\left|\Delta f_{2}\right|-\left|\Delta f_{1}\right|\right) \sum_{i=1}^{n}\left(\frac{1}{K_{p i}}\right)
$$

Given the rating of local load of $P_{\mathrm{n}}$ with a non-folding droop, the largest accumulated error in the power loop is

$$
\max \left(\sum_{i=1}^{n} P_{D E R i}^{*}-P_{\text {Load }}\right)=2 P_{n}
$$

Comparing $(8)(11)$, the reduction of IU size is up to $2 P_{\mathrm{n}}-$ $\left|\Delta f_{M G_{-} \max }\right| \sum_{i=1}^{n}\left(\frac{1}{K_{p i}}\right)$.

\section{CASE STUdy}

To verify the proposed control strategies, case studies are carried out using the benchmark system shown in Fig. 1 with

\begin{tabular}{|c|c|c|}
\hline Components & Parameters & Values \\
\hline \multirow{13}{*}{$\begin{array}{l}\text { DER } \\
\text { converters }\end{array}$} & Power rating (per DER) & $200 \mathrm{kVA}$ \\
\hline & Rated voltage & $415 \mathrm{~V}$ \\
\hline & Rated frequency & $50 \mathrm{~Hz}$ \\
\hline & Switching frequency & $5 \mathrm{kHz}$ \\
\hline & Line inductance $L_{D E R 1,2}$ & $65.8 \mathrm{mH}$ \\
\hline & AC filter capacitor & $66.4 \mathrm{mF}$ \\
\hline & $C_{D E R 1,2}$ & \\
\hline & P droop coefficient $K_{p 1,2}$ & $1 / 125 \mathrm{~Hz} / \mathrm{kW}$ \\
\hline & Q droop coefficient $K_{q 1,2}$ & $3 \mathrm{~V} / \mathrm{kVAr}$ \\
\hline & $\mathrm{P}, \mathrm{Q}$ set points $P_{\mathrm{ref}}, Q_{\mathrm{ref}}$ & $100 \mathrm{~kW}$ \\
\hline & & OK KVAr \\
\hline & Maximum $\Delta f_{\text {MG_max }}$ & $\pm 0.1 \mathrm{~Hz}$ \\
\hline & Folded power $\mathrm{P}_{0}$ & $12.5 \mathrm{~kW}$ \\
\hline \multirow{3}{*}{$\begin{array}{l}\text { DER PI } \\
\text { regulators }\end{array}$} & Voltaeg loop $K_{p}, K_{i}$ & 753,15000 \\
\hline & Current loop $K_{p}, K_{i}$ & 628,98500 \\
\hline & PLL $K_{p}, K_{i}$ & 62,985 \\
\hline \multirow[t]{3}{*}{$\begin{array}{l}\text { Loads and } \\
\text { Line } \\
\text { impedances }\end{array}$} & $\begin{array}{l}\text { Resistive load } \\
\text { Inductive load }\end{array}$ & $\begin{array}{l}0.5 \text { to } 1 \text { p.u. } \\
\text { with power factor } \\
1 \text { to } 0.9 \text {, } \\
\text { respectively }\end{array}$ \\
\hline & Line impedance $1 L_{l 1}$ & $8 \%$ p.u. \\
\hline & Line impedance $2 L_{l 2}$ & $5 \%$ p.u. \\
\hline \multirow{5}{*}{$\begin{array}{l}\text { IU BTB } \\
\text { VSCs }\end{array}$} & Rated power & $40 \mathrm{~kW}$ \\
\hline & AC voltage & $415 \mathrm{~V}$ \\
\hline & DC voltage & $\pm 400 \mathrm{~V}$ \\
\hline & Inductance $L_{I U 1,2}$ & $65.8 \mathrm{mH}$ \\
\hline & Capacitor $C_{I U 1,2}$ & $66.4 \mathrm{mF}$ \\
\hline
\end{tabular}
MATLAB/Simulink. The two DERs are simulated as identical VSCs connected to constant DC voltage. An ideal harmonic free infinite bus is used to model the utility grid. The loads are inductive. The parameters of the system are specified in Table I. Three operational cases are considered in this section.

Table I. Parameters of the proposed system

\section{A. Islanded operation and reconnection test}

An islanded and reconnection operation test is carried out and the Table II shows the sequences. Figs. 6 and 7 compare the system response between the cases without and with the proposed folded $P-f$ droop scheme. 
Table II Event sequence for Case A

\begin{tabular}{c|c|c|c|c}
\hline Sequence & (1) & (2) & (3) & (4) \\
\hline Event & $\begin{array}{c}\text { Load step } \\
200 \mathrm{~kW}\end{array}$ & $\begin{array}{c}\text { Start Re- } \\
\text { synchronization }\end{array}$ & $\begin{array}{c}\mathrm{CB} \\
\text { re-closed }\end{array}$ & $\begin{array}{c}\text { Power transfer } \\
\text { from IU to IU- } \\
\mathrm{CB}\end{array}$ \\
\hline Time(1) & $0.5 \mathrm{~s}$ & $2 \mathrm{~s}$ & $5 \mathrm{~s}$ & $5.5 \mathrm{~s}$ \\
\hline Time(2) & $0.5 \mathrm{~s}$ & $1.5 \mathrm{~s}$ & $3 \mathrm{~s}$ & $3.5 \mathrm{~s}$ \\
\hline
\end{tabular}
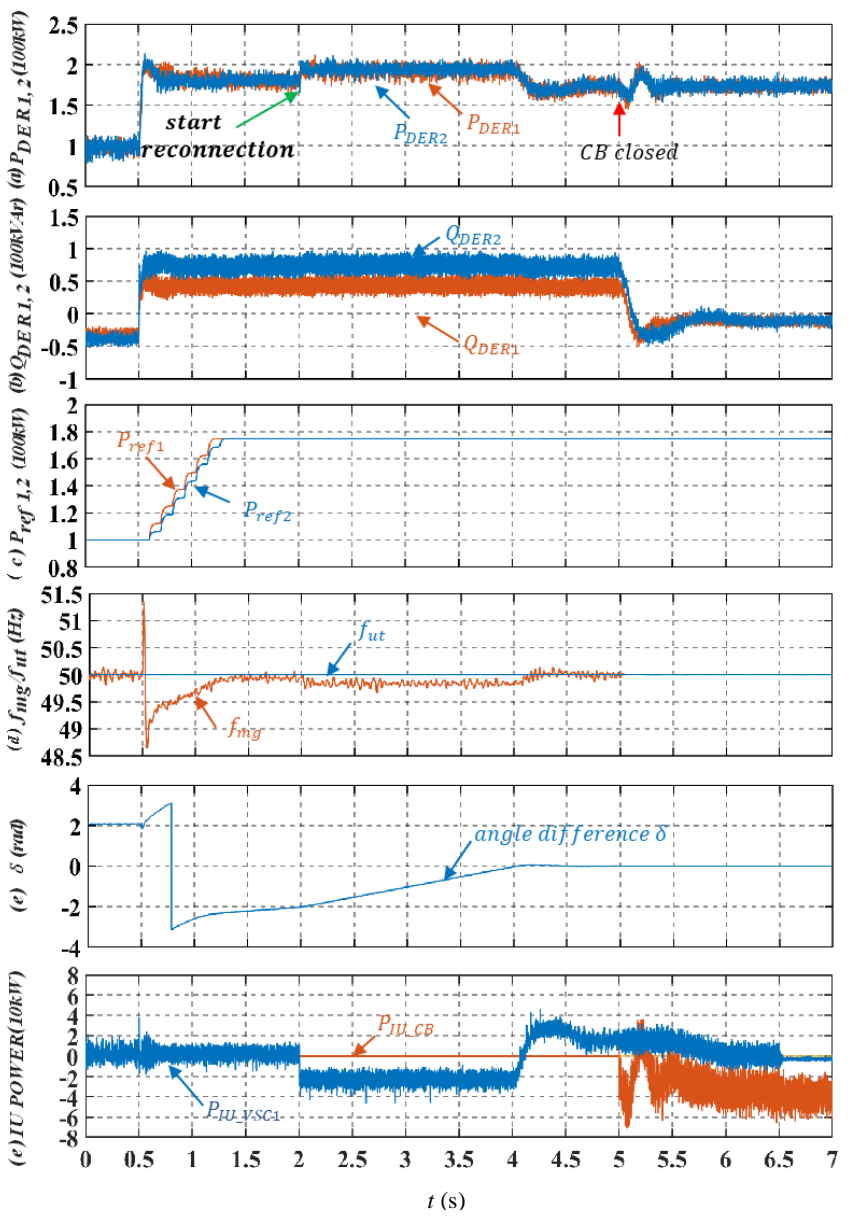

Fig. 6. Islanded operation and Reconnecuon with folded droop (a)DER1,2 Active power; (b) DER1,2 Reactive power; (c) DER1,2 Reference active power; (d) Frequency of MG and Utility grid; (e) Angle difference between MG and Utility grid; (f) Active power of IU-VSC and IU-CB.

The system starts in islanded operation with IU-CB open and IU-VSCs blocked, and an initial load of $200 \mathrm{~kW}$ (0.5 p.u.) is connected. From Fig. 6(a) and Fig. 7(a), it can be seen that the power outputs of both DERs $\left(P_{D E R I}\right.$ and $\left.P_{D E R 2}\right)$ are almost identical at $100 \mathrm{~kW}$ for both cases. The deviations of frequency from $50 \mathrm{~Hz}$ are nearly zero at the start of Fig. 6(d) and Fig. 7(c) for both schemes, which corresponds to the droop and active power reference values $\left(1 / K_{p l, 2}=125 \mathrm{~kW} / \mathrm{Hz}\right.$ and $\left.P_{\text {DER } 1,2}{ }^{*}=100 \mathrm{~kW}\right)$.

As indicated by Table II, at $t=0.5 \mathrm{~s}$, the MG load steps up from a pure resistive load $200 \mathrm{~kW}$ to an inductive load of 400 kVA with a power factor of 0.9. As seen in Fig. 6(a) and Fig. 7 (a), DERs in both cases step up to approximately $185 \mathrm{~kW}$ simultaneously. The frequency of the $\mathrm{MG}$ drops to approximately $49.3 \mathrm{~Hz}$ according to the conventional P-f droop as shown in Fig. 7(c). Whereas, for the results shown in Fig. 6, folded droop is activated. That is, the reference power of each
DER is folded once the frequency deviation is greater than $\Delta f_{\text {MG }_{-} \text {max }}=0.1 \mathrm{~Hz}$. As shown In Fig. 6(c), the active power set point of DER 1 and 2 is increased from initial value of $100 \mathrm{kw}$ to $185 \mathrm{~kW}$ so $f_{M G}$ is restored to approximately $49.95 \mathrm{~Hz}$ in Fig. 6(d).

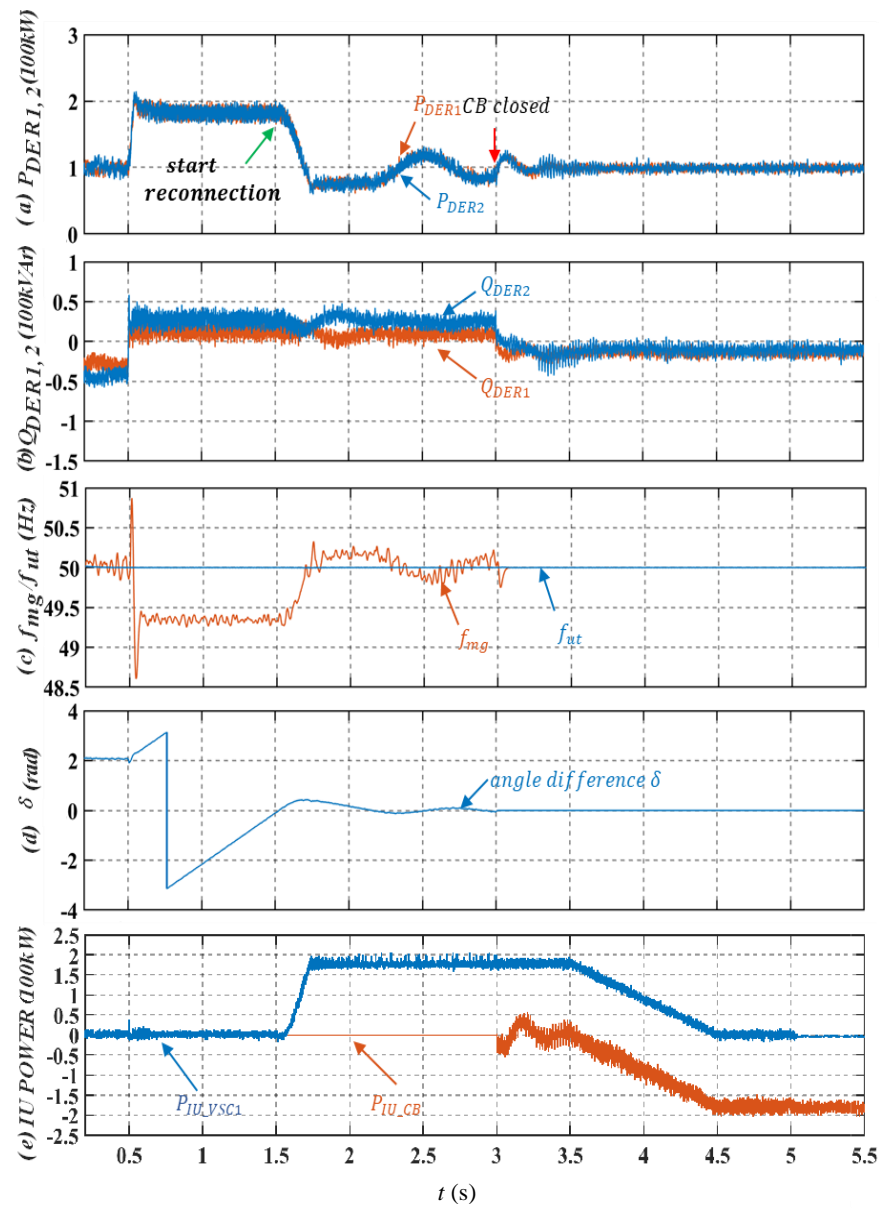

Fig. 7. Islanded operation and reconnection without folded droop (a) DER1,2 Active power ;(b) DER1,2 Reactive power;(c) MG frequency and Utility grid frequency;(d) Angle difference between MG system and the Utility grid;(e) Active power of IU-VSC and IU-CB.

At $t=1.5 \mathrm{~s}$ and $2 \mathrm{~s}$, the resynchronization process is initiated by the IU in Fig. 6 and Fig. 7, respectively. With the proposed synchronizing method demonstrated by Fig. 2, the angle between the MG and the mains starts to reduce immediately after the process starts in both cases.

With the normal droop design, to shift the MG frequency from $49.3 \mathrm{~Hz}(\Delta f \approx 0.7)$ to the grid frequency of $50 \mathrm{~Hz}$, the IU $\left(P_{I U_{-} V S C l}\right)$ delivers a power approximately $175 \mathrm{~kW}$ to the $\mathrm{MG}$ as seen in Fig. 7(e) at $t=3 \mathrm{~s}$. According to (8), the conventional droop should demand a minimum IU power of $80 \mathrm{~kW}+80 \mathrm{~kW}$ $=160 \mathrm{~kW}$ to synchronize with grid frequency. Whereas in the simulation of Fig. 7, the operational power is up to approximately $175 \mathrm{~kW}$. In the simulated operation, the operational frequency deviation is up to $0.2 \mathrm{~Hz}$, which accelerates the tracking of grid angle but demand extra power capacity.

In contrast, the magnitude of IU power with the folded droop scheme is capped approximately at $25 \mathrm{~kW}$. This is achieved by setting the DER d-axis saturation corresponding to 
this rating to allow a maximum of $0.1 \mathrm{~Hz}$ frequency deviation from the grid frequency of $50 \mathrm{~Hz}$. Assigning $\Delta f_{M G_{-} \max }=$ $0.1 \mathrm{~Hz}, \Delta f_{\text {grid_max }}=0$ and $K_{p 1}=K_{p 2}=\frac{1}{125} \mathrm{~Hz} / \mathrm{kW}$ to $(12)$, the essential power to synchronize the frequencies is $25 \mathrm{~kW}$, which corresponds well with Fig. 6. This effective synchronization is further evidenced by the maintained angular difference at $\delta=0 \mathrm{rad}$ after $\mathrm{t}=4.5 \mathrm{~s}$ in Fig. 6(e).

After the IU finds the close of IU-CB successful, the power delivered through IU-VSCs is gradually taken over by IU-CB in both cases of Figs. 6 and 7 from $t=5 \mathrm{~s}$ and $3 \mathrm{~s}$, respectively. IU-VSCs are then blocked and isolated to start grid-connected mode.

The power reduction of IU-VSC is $175-25=150 \mathrm{~kW}$ for this scenario by introducing folded droop. Since the minimum power capacity for re-synchronization increase with $\left(\left|\sum_{i=1}^{n} P_{D E R i}^{*}-P_{\text {Load }}\right|\right)$ according to (8), this reduction is expected to be more prominent with greater step of active power after an islanding operation.

Throughout this process of islanded load switching and reconnection, all the actions are taken with local detections only. Thus, fast communications are not needed. Despite of identical droop gains in frequency regulators, the folded droop significantly reduces the MG frequency derivation and curtail the essential power for synchronization and consequently reduce the power capacity of the IU converter.

\section{B. Planned islanding operation}

Table III Event sequence for Case B

\begin{tabular}{c|c|c|c|c}
\hline Sequence & (1) & (2) & (3) & (4) \\
\hline Event & $\begin{array}{c}\text { Start islanding } \\
\text { transition }\end{array}$ & Activate IU & Open CB & Block IU \\
\hline Time & $0 \mathrm{~s}$ & $0.25 \mathrm{~s}$ & $1 \mathrm{~s}$ & $2 \mathrm{~s}$ \\
\hline
\end{tabular}

For the islanding operation based on the IU system, this process is a reverse version of reconnection scheme. As a planned islanding action, the limitation is mainly due to the rating of IU-VSCs. If the power transmitted through the IU-CB prior to the planned islanding is smaller than the IU-VSCs rating, all the power in IU-CB can be shifted to IU-VSCs before the opening of IU-CB so as to achieve smoothly transition. Otherwise, IU-CB must be opened immediately after a grid event and cause significant transient variations to the MG.

Considering the rating of IU-VSCs is sufficient and the operational sequence in Table III, Fig. 8 shows the simulation results during planned islanding. From $t=0$ to $0.25 \mathrm{~s}$, the MG and the mains are inter-connected through the IU-CB with $40 \mathrm{~kW}$ power transferring from the mains, which is shown in Fig. 8 (e). At 0.25 s, IU-VSC 1 is activated and starts increasing its power export to the MG. At $0.75 \mathrm{~s}$, the power carried by IUCB drops to zero and IU-CB is finally opened at $1 \mathrm{~s}$. The MG is now only connected to the main through IU-VSCs providing 40 $\mathrm{kW}$ to the MG. At $1.25 \mathrm{~s}$, the output power of IU-VSC1 starts to ramp down to zero within $0.5 \mathrm{~s}$. As a result, from $t=1.75 \mathrm{~s}$, the MG is completely supplied by DER 1 and 2 . A frequency deviation of $0.2 \mathrm{~Hz}$ is observed in Fig. 8(d) thereafter. At $t=2$ $\mathrm{s}$, the IU-VSCs are blocked and MG becomes an island.

Comparing with the unplanned cases shown in Section AFigs. 6 and 7, the planned islanding operation can bring about a significant reduction in the variation of frequency during the transition by $86 \%$ (from $1.4 \mathrm{~Hz}$ down to $0.2 \mathrm{~Hz}$ ) while the net loads within the MG are identical. However, this is at a cost of IU-rating to fully accommodate the net load, which means a trade-off between the allowance of net load and the size of IU must be considered in the plan of operation.
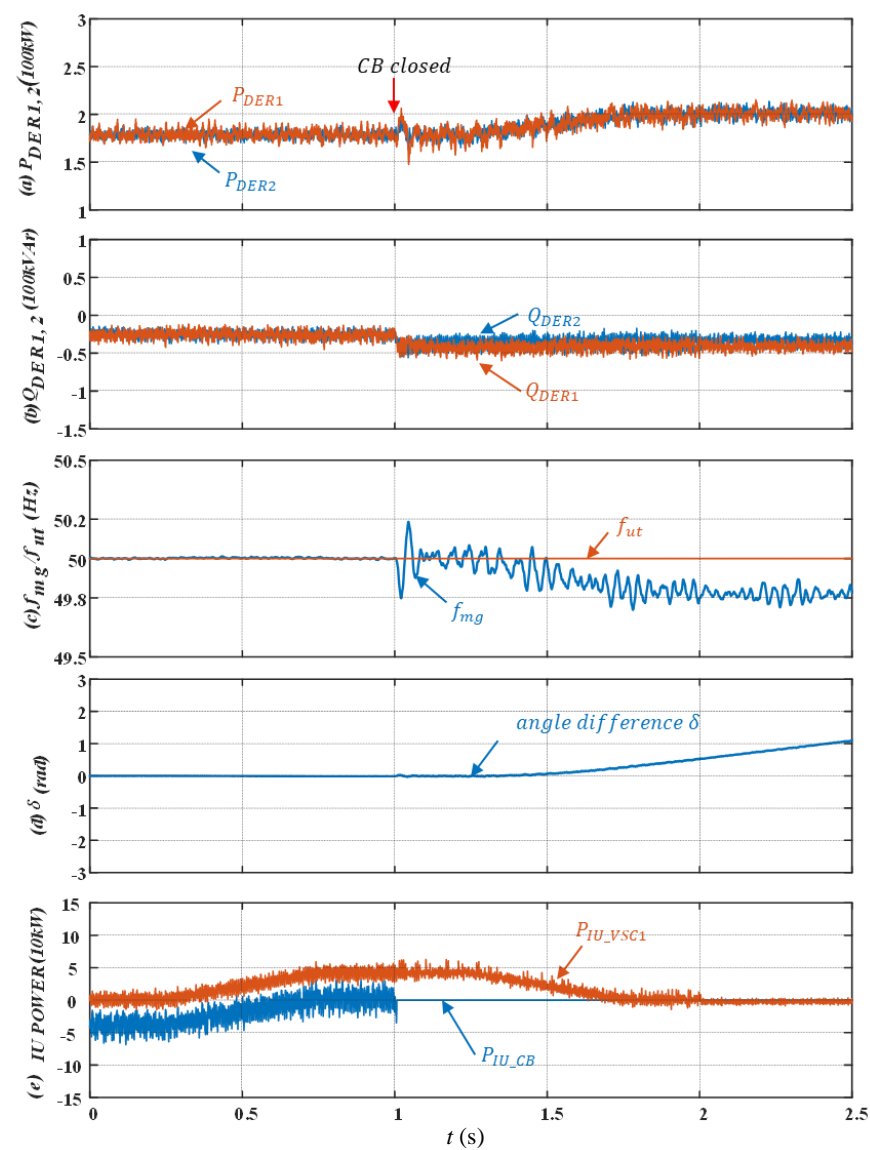

Fig. 8. Planned islanding operation

(a)DER1,2 active power ;(b) DER1,2 reactive power;(c) MG frequency and Utility grid frequency;(d) Angle difference between MG system and the Utility grid;(e) Active power of IU-VSC and IU-CB.

\section{CONCLUSIONS}

By paralleling a mechanical circuit breaker IU-CB and back-to-back VSCs, an IU can effectively enable autonomous transitions between grid-connected and islanded modes with minimum requirement of communications and seamless power supply, provided mutli-loop cascaded DER controls are in place.

With the proposed folded droop and planned islanding operation scheme, the frequency deviation at steady state and during an islanding transition can be effectively reduced, respectively. By manipulating the parametric design of the folded droop, the power capacity of IUs can be capped to a predefined level. However, as a trade-off, the reduction of power capacities of IU converter will compromise the effect in suppressing the transient frequency deviation during a planned islanding operation. 


\section{REFERENCES}

[1] C. T. Lee, R. P. Jiang, and P. T. Cheng, "A grid synchronization method for droop-controlled distributed energy resource converters," IEEE Trans. Ind. Appl., vol. 49, no. 2, pp. 954-962, 2013.

[2] J. Rocabert, A. Luna, F. Blaabjerg, and P. Rodríguez, "Control of power converters in AC microgrids," IEEE Trans. Power Electron., vol. 27, no. 11, pp. 4734-4749, 2012.

[3] J. A. P. Lopes, C. L. Moreira, and A. G. Madureira, "Defining control strategies for analysing microgrids islanded operation," 2005 IEEE Russ. Power Tech, PowerTech, vol. 21, no. 2, pp. 916-924, 2005.

[4] I. J. Balaguer, Q. Lei, S. Yang, U. Supatti, and F. Z. Peng, "Control for grid-connected and intentional islanding operations of distributed power generation," IEEE Trans. Ind. Electron., vol. 58, no. 1, pp. 147-157, 2011.

[5] L. G. Meegahapola, D. Robinson, A. P. Agalgaonkar, S. Perera, and P. Ciufo, "Microgrids of commercial buildings: Strategies to manage mode transfer from grid connected to islanded mode," IEEE Trans. Sustain. Energy, vol. 5, no. 4, pp. 1337-1347, 2014.

[6] W. Xu, G. Zhang, C. Li, W. Wang, G. Wang, and J. Kliber, "A power line signaling based technique for anti-islanding protection of distributed generators - Part I: Scheme and analysis," IEEE Trans. Power Deliv., vol. 22, no. 3, pp. 1758-1766, 2007.

[7] C. Buque, S. Chowdhury, and S. P. Chowdhury, "Modelling and simulation of reverse power relay for loss of mains protection of distributed generation in microgrids," IEEE Power Energy Soc. Gen. Meet., pp. 1-5, 2013.

[8] S. Il Jang and K. H. Kim, "An islanding detection method for distributed generations using voltage unbalance and total harmonic distortion of current," IEEE Trans. Power Deliv., vol. 19, no. 2, pp. 745-752, 2004.

[9] M. N. Arafat, A. Elrayyah, and Y. Sozer, "An effective smooth transition control strategy using droop based synchronization for parallel inverters," 2014 IEEE Energy Convers. Congr. Expo. ECCE 2014, vol. 51, no. 3, pp. 3964-3970, 2014.

[10] A. Pina Martins, A. S. Carvalho, and A. S. Araujo, "Design and implementation of a current controller for the parallel operation of standard UPSs," IECON Proc. (Industrial Electron. Conf., vol. 1, pp. 584-589, 1995.

[11] J. M. Guerrero, J. C. Vasquez, J. Matas, L. G. De Vicuña, and M. Castilla, "Hierarchical control of droop-controlled AC and DC microgrids - A general approach toward standardization," IEEE Trans. Ind. Electron., vol. 58, no. 1, pp. 158-172, 2011.

[12] A. B. Shitole et al., "Grid Interfaced Distributed Generation System with Modified Current Control Loop Using Adaptive Synchronization Technique," IEEE Trans. Ind. Informatics, vol. 13, no. 5, pp. 2634-2644, 2017.

[13] N. R. Merritt, C. Chakraborty, and P. Bajpai, "New Voltage Control Strategies for VSC-Based DG Units in an Unbalanced Microgrid," IEEE Trans. Sustain. Energy, vol. 8, no. 3, pp. 1127-1139, 2017.

[14] J. Kim, J. M. Guerrero, P. Rodriguez, R. Teodorescu, and K. Nam, "Mode adaptive droop control with virtual output impedances for an inverter-based flexible AC microgrid," IEEE Trans. Power Electron., vol. 26, no. 3, pp. 689-701, 2011.

[15] Y. A. R. I. Mohamed, H. H. Zeineldin, M. M. A. Salama, and R. Seethapathy, "Seamless formation and robust control of distributed generation microgrids via direct voltage control and optimized dynamic power sharing," IEEE Trans. Power Electron., vol. 27, no. 3, pp. 12831294, 2012.

[16] F. Guo, C. Wen, J. Mao, and Y. D. Song, "Distributed Secondary Voltage and Frequency Restoration Control of Droop-Controlled Inverter-Based Microgrids," IEEE Trans. Ind. Electron., vol. 62, no. 7, pp. 4355-4364, 2015.

[17] J. W. Simpson-Porco, Q. Shafiee, F. Dorfler, J. C. Vasquez, J. M.
Guerrero, and F. Bullo, "Secondary Frequency and Voltage Control of Islanded Microgrids via Distributed Averaging," IEEE Trans. Ind. Electron., vol. 62, no. 11, pp. 7025-7038, 2015.

[18] G. Chen and Z. Guo, "Distributed secondary and optimal active power sharing control for islanded microgrids with communication delays," IEEE Trans. Smart Grid, vol. 10, no. 2, pp. 2002-2014, 2019.

[19] K. De Brabandere et al., "A Voltage and Frequency Droop Control Method for Parallel Inverters," IEEE Trans. Power Electron., vol. 22, no. 4, pp. 1107-1115, 2007.

[20] J. A. Suul, S. D’Arco, and G. Guidi, "Virtual Synchronous MachineBased Control of a Single-Phase Bi-Directional Battery Charger for Providing Vehicle-to-Grid Services," IEEE Trans. Ind. Appl., vol. 52, no. 4, pp. 3234-3244, 2016.

[21] L. Li, Y. Sun, Z. Liu, X. Hou, G. Shi, and M. Su, "A Decentralized Control with Unique Equilibrium Point for Cascaded-Type Microgrid," IEEE Trans. Sustain. Energy, vol. 10, no. 1, pp. 324-326, 2019.

[22] M. Gao, M. Chen, B. Zhao, B. Li, and S. Member, "Design of Control System for Smooth Mode-Transfer of Grid-Tied Mode and Islanding Mode in Microgrid," vol. 35, no. 6, pp. 6419-6435, 2020.

[23] Y. W. Li and C. N. Kao, "An accurate power control strategy for powerelectronics-interfaced distributed generation units operating in a lowvoltage multibus microgrid," IEEE Trans. Power Electron., vol. 24, no. 12, pp. 2977-2988, 2009.

[24] H. Sekhavatmanesh and S. Member, "Power and Voltage Control in a Grid-Connected Microgrid System with a Back-to-Back Converter," no. February, pp. 3-4, 2015.

[25] J. C. Vasquez, J. M. Guerrero, A. Luna, P. Rodríguez, and R. Teodorescu, "Adaptive droop control applied to voltage-source inverters operating in grid-connected and islanded modes," IEEE Trans. Ind. Electron., vol. 56, no. 10, pp. 4088-4096, 2009.

[26] Y. A. R. I. Mohamed and E. F. El-Saadany, "Adaptive decentralized droop controller to preserve power sharing stability of paralleled inverters in distributed generation microgrids," IEEE Trans. Power Electron., vol. 23, no. 6, pp. 2806-2816, 2008.

[27] J. M. Guerrero, J. C. Vásquez, J. Matas, M. Castilla, and L. García de Vicuna, "Control strategy for flexible microgrid based on parallel lineinteractive UPS systems," IEEE Trans. Ind. Electron., vol. 56, no. 3, pp. 726-736, 2009.

[28] L. Yu, R. Li, and L. Xu, "Distributed PLL-Based Control of Offshore Wind Turbines Connected With Diode-Rectifier-Based HVDC Systems," vol. 33, no. 3, pp. 1328-1336, 2018.

[29] I. Y. Chung, W. Liu, D. A. Cartes, E. G. Collins, and S. Il Moon, "Control methods of inverter-interfaced distributed generators in a microgrid system," IEEE Trans. Ind. Appl., vol. 46, no. 3, pp. 10781088, 2010.

[30] M. N. Arafat, A. Elrayyah, and Y. Sozer, "An effective smooth transition control strategy using droop based synchronization for parallel inverters," 2014 IEEE Energy Convers. Congr. Expo. ECCE 2014, vol. 51, no. 3, pp. 3964-3970, 2014.

[31] L. Xu, L. Yao, and C. Sasse, "Grid integration of large DFIG-based wind farms using VSC transmission," IEEE Trans. Power Syst., vol. 22, no. 3, pp. 976-984, 2007.

[32] M. Naderi, Y. Khayat, Q. Shafiee, T. Dragicevic, H. Bevrani, and F. Blaabjerg, "Interconnected Autonomous ac Microgrids via Back-toBack Converters-Part II: Stability Analysis," IEEE Trans. Power Electron., vol. 35, no. 11, pp. 11801-11812, 2020.

[33] X. Wu, C. Shen, and R. Iravani, "A Distributed, Cooperative Frequency and Voltage Control for Microgrids," IEEE Trans. Smart Grid, vol. 9, no. 4, pp. 2764-2776, 2018.

[34] L. Luo and S. V. Dhople, "Spatiotemporal model reduction of inverterbased islanded microgrids," IEEE Trans. Energy Convers., vol. 29, no. 4, pp. 823-832, 2014. 
APPENDIX I COMPARISON OF TYPICAL TRANSITION SCHEME OF A MICROGRID

\begin{tabular}{|c|c|c|c|}
\hline & Transition smoothness & Capital cost of interface & $\begin{array}{c}\text { Interface Efficiency at } \\
\text { Steady State }\end{array}$ \\
\hline $\begin{array}{c}\text { Mode-switching based } \\
\text { Scheme without PCC } \\
\text { interface converter [23] }\end{array}$ & low & low & high \\
\hline $\begin{array}{c}\text { PCC Interface with BtB } \\
\text { converter scheme [24] }\end{array}$ & high & high & high \\
\hline $\begin{array}{c}\text { Cascaded control without } \\
\text { PCC interface converter } \\
\text { [8][9][25] }\end{array}$ & $\begin{array}{c}\text { median in islanding. } \\
\text { low in re-connecting. } \\
\text { inflexible in planned } \\
\text { islanding. }\end{array}$ & low & high \\
\hline $\begin{array}{c}\text { The proposed scheme of } \\
\text { folding droop and IU } \\
\text { configuration }\end{array}$ & high & $\begin{array}{c}\text { Median } \\
\text { Can be reduced by trade- } \\
\text { off of flexibility in planned } \\
\text { islanding. }\end{array}$ & \\
\hline
\end{tabular}

